\title{
Stabilization mechanism of edge states in graphene
}

\author{
K. Sasaki, ${ }^{1, *}$ S. Murakami, ${ }^{2}$ and R. Saito ${ }^{3}$ \\ ${ }^{1}$ Institute for Materials Research, Tohoku University, Sendai 980-8577, Japan \\ ${ }^{2}$ Department of Applied Physics, University of Tokyo, Hongo, Bunkyo-ku, Tokyo 113-8656, Japan \\ ${ }^{3}$ Department of Physics, Tohoku University and CREST, JST, Sendai 980-8578, Japan
}

(Dated: Received 29 July 2005; accepted 23 January 2006)

\begin{abstract}
It has been known that edge states of a graphite ribbon are zero-energy, localized eigenstates. We show that next nearest-neighbor hopping process decreases the energy of the edge states at zigzag edge with respect to the Fermi energy. The energy reduction of the edge states is calculated analytically by first-order perturbation theory and numerically. The resultant model is consistent with the peak of recent scanning tunneling spectroscopy measurements.
\end{abstract}

Carbon-based materials have attracted much attention from various points of view $\stackrel{1}{\underline{1}}$ In particular, their electrical properties are of great interest, where topology plays an important role because it is relevant for a rich variety of the electronic properties. As one topological aspect, boundaries can induce localized states called edge states $^{2.3}$ at graphite edge. Theoretically, edge states are zero-energy eigenstates relative to the Fermi energy and are predicted to make a certain magnetic ordering. 2.4 Experimentally, by scanning tunneling microscopy (STM) and spectroscopy (STS) of graphite edge, a peak in the local density of states (LDOS) has been observed, and

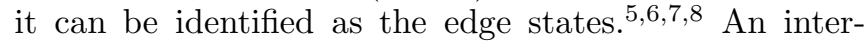
esting point is that the peak is located not just at the Fermi energy but below the Fermi energy by about 20 $\mathrm{meV}$. 5.6 Since several possible perturbations would shift the energy eigenvalue above the Fermi energy, $\underset{7.8}{7 t}$ is not a simple problem to find a consistent perturbation that can decrease or stabilize the energy of the edge state. In this letter we show that the next nearest-neighbor (NNN) hopping process is a key factor which decreases the energy eigenvalue of the edge state. This is shown by the first order perturbation theory for the tight-binding Hamiltonian and by numerical energy band structure calculation.

First, we explain the energy band structures for the graphene, using the nearest-neighbor tight-binding Hamiltonian for the honeycomb lattice with the hopping integral $-\gamma_{0}(\sim-3 \mathrm{eV})$. We ignore the electron spin for simplicity. The eigenenergies of this model are given by $\pm \gamma_{0}|f(\mathbf{k})|$ where $f(\mathbf{k}) \equiv \sum_{a=1,2,3} e^{i \mathbf{k} \cdot \mathbf{R}_{a}}$ and $\mathbf{R}_{a}$ $(a=1,2,3)$ are the vectors from an A-sublattice site to the neighboring B-sublattice sites. The two energy bands are degenerate at the two $\mathbf{k}$ points called the $\mathrm{K}$ and $\mathrm{K}^{\prime}$ points. Now we consider a zigzag nanotube illustrated in Fig. 1. Henceforth we define coordinate axes around and along the nanotube axis as $x_{1}$ and $x_{2}$. In a zigzag nanotube (of finite length) the dimensionless wave vector around the tube, $q \equiv \mathbf{k} \cdot \mathbf{a}_{1}=\sqrt{3} k_{1} a_{\mathrm{cc}}$, remains a good quantum number and is now quantized as an integer multiple of $2 \pi / n$ with the chiral vector $C_{h}=(n, 0)$. For $n \rightarrow \infty$ the model represents a graphite ribbon with zigzag edges discussed in Ref. 2. The delocalized eigenstates for the nanotube are similar to those of the graphene. We take the Brillouin zone to be $0 \leq q<2 \pi$. The states at the Fermi level without doping are characterized by $f(\mathbf{k})=0$, which implies $q=2 \pi / 3,4 \pi / 3$, corresponding to the $\mathrm{K}$ and $\mathrm{K}^{\prime}$ points, respectively.

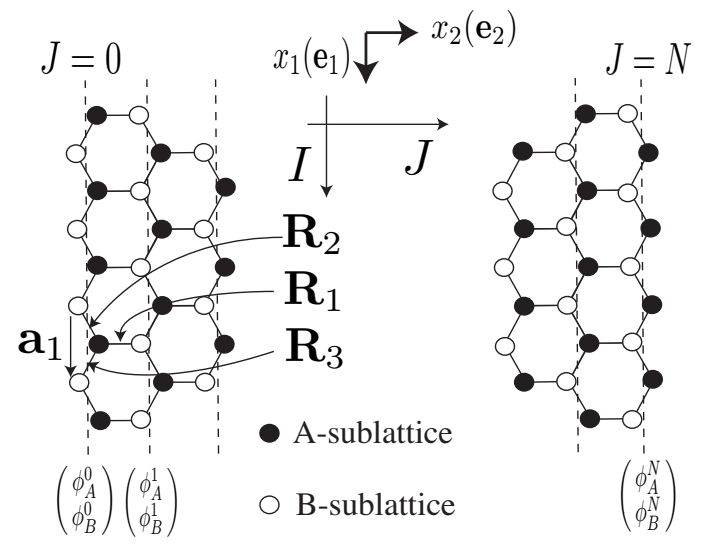

FIG. 1: Lattice structure of a zigzag carbon nanotube with finite length. The filled (open) circle indicates the A sublattice (B sublattice). Both the left and right edges are zigzag edges. $\mathbf{a}_{1} \equiv \sqrt{3} a_{\mathrm{cc}} \mathbf{e}_{1}$ is the primitive vector around tubule axis where $a_{\mathrm{cc}}$ denotes the carbon-carbon bond length and $\mathbf{e}_{1}\left(\mathbf{e}_{2}\right)$ is the unit vector around (along) the tubule axis.

On the other hand, as we noted in the introduction, the zigzag nanotube has edge states in addition to delocalized (bulk) states. Whether or not such edge states are allowed depends on the boundary conditions. To analyze the edge states, we consider the eigenvalue equation

$$
\begin{aligned}
& -E \phi_{A}^{J}=\gamma_{0} \phi_{B}^{J+1}+G \phi_{B}^{J}, \\
& -E \phi_{B}^{J+1}=\gamma_{0} \phi_{A}^{J}+G \phi_{A}^{J+1}, \quad(J=0, \ldots, N-1)
\end{aligned}
$$

where $G \equiv 2 \gamma_{0} \cos (q / 2)$ and ${ }^{t}\left(\phi_{A}^{J}, \phi_{B}^{J}\right)$ is the wave function at $J$ th site (see Fig. 1); the wave function at site $(I, J)$ of A sublattice (B sublattice) can be written as $\psi_{(A, B) I}^{J}(q)=\exp (i I q) \phi_{(A, B)}^{J}(q)$.

The energy eigenvalues for the nanotube with zigzag edges can be calculated by imposing the boundary condition: $-E \phi_{A}^{N}=G \phi_{B}^{N}$ and $-E \phi_{B}^{0}=G \phi_{A}^{0}$. This boundary condition for the tube supports edge states around the Fermi energy $\stackrel{2}{2}$ By direct calculation one finds that the 
edge states exist if $|G|<\gamma_{0}$, implying $2 \pi / 3<q<4 \pi / 3$, and the wave function of the edge state for $-\gamma_{0}<G<0$ is

$$
\begin{aligned}
\phi_{A}^{J} & =\left[\frac{\sinh (J+1) \varphi}{\sinh \varphi}\right] \phi_{A}^{0}, \\
\phi_{B}^{J} & =\left[\frac{\gamma_{0}}{G} \frac{\sinh J \varphi}{\sinh \varphi}+\frac{\sinh (J+1) \varphi}{\sinh \varphi}\right] \phi_{B}^{0}, \quad(J=0, \ldots, N)
\end{aligned}
$$

where $\varphi$ is a positive number satisfying $\gamma_{0} \sinh (N+$ 1) $\varphi+G \sinh (N+2) \varphi=0$ and depends on the wave vector around the tube via the boundary condition: $e^{-\varphi} \approx-2 \cos (q / 2)$. The energy eigenvalue is $E=$ $\pm \gamma_{0} \sinh \varphi / \sinh (N+2) \varphi$, which is exponentially close to the Fermi level. When $\varphi$ is large, the edge state asymptotically behaves exponentially near the edges

$$
\begin{aligned}
& 0 \lesssim J \ll N: \phi_{A}^{J} \sim 0, \quad \phi_{B}^{J} \sim \phi_{B}^{0} e^{-J \varphi}, \\
& 0 \ll J \lesssim N: \phi_{A}^{J} \sim \phi_{A}^{N} e^{(J-N) \varphi}, \quad \phi_{B}^{J} \sim 0,
\end{aligned}
$$

where $\phi_{B}^{0}=\mp \phi_{A}^{N}$. The localization length is given by $\varphi^{-1}\left(3 a_{\mathrm{cc}} / 2\right)$. The energy eigenvalues are $E \approx$ $\pm \gamma_{0} e^{-N \varphi} \approx 0$. For $0<G<\gamma_{0}$, the edge state wave function is obtained by multiplying $(-1)^{J}$ by $\phi_{A}^{J}$ and $\phi_{B}^{J}$ in Eq. (2) with $G \rightarrow-G$. When approaching $G \rightarrow \pm \gamma_{0}$, i.e., $q \rightarrow 2 \pi / 3,4 \pi / 3$, the localization length becomes infinite, and the edge state finally becomes a bulk state. In this sense, the zero-energy states at $q=2 \pi / 3,4 \pi / 3$ can be called critical states ${ }^{\frac{9}{2}}$ On the other hand, the state with $q=\pi$ corresponds to $\varphi=\infty$; it is the most localized state, which has nonzero amplitude only at the edge sites.

Thus far we have considered the nearest-neighbor hopping process only. Now, we include the NNN hopping process with hopping integral $-\gamma_{n}$. We show that the inclusion of the NNN hopping decreases the edge state energy with respect to the Fermi level.

First, we prove this by means of the first-order perturbation theory. The Hamiltonian matrix reads as

$$
\mathcal{H}=\left(\begin{array}{cc}
-\gamma_{n}\left(|f(\mathbf{k})|^{2}-3\right) & -\gamma_{0} f^{*}(\mathbf{k}) \\
-\gamma_{0} f(\mathbf{k}) & -\gamma_{n}\left(|f(\mathbf{k})|^{2}-3\right)
\end{array}\right) .
$$

The new energy dispersion relation is then given by

$$
E(\mathbf{k})= \pm \gamma_{0}|f(\mathbf{k})|-\gamma_{n}|f(\mathbf{k})|^{2}+3 \gamma_{n} .
$$

Henceforth we subtract the constant term $3 \gamma_{n}$ in the energy $E(\mathbf{k})$. Thus the energy shift due to the NNN hopping is given by $\Delta E=-\gamma_{n}|f(\mathbf{k})|^{2}$. The critical states have $f(\mathbf{k})=0$, which yields $\Delta E=0$. The edge states appearing in the zigzag nanotubes with long length correspond to $f(\mathbf{k}) \approx 0$ with complex wave vector along the nanotube $k_{2}\left(\equiv \mathbf{k} \cdot \mathbf{e}_{2}\right)$. This naïvely leads to the result that the energy shift, $\Delta E$, of the edge states due to the NNN hopping is zero. Nevertheless, it is not correct, as we will see here; the existence of the boundaries is crucial for the calculation of energy shift. The resulting shift will turn out to be negative, thus stabilizing the edge states.

Within the first-order perturbation theory, the energy shift of the edge state is given by the expectation value of the NNN hopping with respect to the edge state. As a simple example, we consider the energy shift for the most localized edge state $(q=\pi)$. The wave function of this edge state is given by $\psi_{B I}^{0}=\psi_{A I}^{N}=(-1)^{I} / \sqrt{2 n}$ and zero otherwise. The energy shift is negative and is given by $-2 \gamma_{n} \cos q-3 \gamma_{n}=-\gamma_{n}$. In the similar way, one can evaluate the energy shift for general edge states as a function of $q$. From the asymptotic behavior of the wave function given by Eq. (3), we evaluate the energy shift to the first-order in the NNN hopping as

$$
\Delta E \approx \gamma_{n}\left(-1+G^{2} / \gamma_{0}^{2}\right)=\gamma_{n}(2 \cos q+1) .
$$

In particular, for the critical states, it reproduces $\Delta E=0$ as mentioned previously. Among the localized states, the negative energy shift, $|\Delta E|=-\Delta E$, is largest for the most localized state.

To confirm these results, we numerically diagonalize the tight-binding Hamiltonian for a graphite ribbon with zigzag edges. We consider the energy band structure of a graphite ribbon with $N=20$. In Fig. 2(a), we show the energy band structure without the NNN hopping: $\gamma_{n}=0$. The edge states form a flatband at $E=0^{2}$ which makes a peak in the LDOS shown in Fig. 2(c). In Fig. 2(b), we plot the energy band structure including the NNN hopping. We set $\gamma_{n}=0.1 \gamma_{0}{ }^{10}$ which shifts the Fermi level to $E_{F}=0.3 \gamma_{0} \stackrel{11}{\underline{1}}$ The critical states are located at $E_{F}\left(=3 \gamma_{n}\right)$, and the edge states have lower energies by $-\gamma_{n} \leq \Delta E<0$, in agreement with Eq. (6). Therefore, we conclude that the energy minimum at $q=$ $\pi$ shown in Fig. 2(b) gives a sharp peak in the LDOS. In Fig. 2(d) we plot the LDOS at several points near the zigzag edge as a function of energy measured from the shifted Fermi level. The abovementioned peak is clearly seen and this is responsible for the peak in the LDOS observed by recent experiments $\frac{5.6}{.6}$

It is noted that our model does not include the overlapping integral ( $s$ parameter $\left.{ }^{1}\right)$ which increases (decreases) the conduction (valence) band width. To examine the effect of $s$ parameter on the edge states, we performed the energy band structure calculation in an extended tightbinding framework ${ }^{12}$ and found the similar behavior of the energy band structure depicted in Fig. 22 (b). The most stable edge state is $q=\pi$ and the energy eigenvalue is located below the Fermi level by about $\gamma_{n}$. The $s$-parameter does not affect the energy spectrum near the Fermi level since the effect of the overlapping integral is proportional to the energy of the corresponding states measured from the Fermi level.

Here, we discuss the relationship between our work and experimental results ${ }^{5.6 .7}$ on a peak of LDOS at graphite edges. Niimi et $a l^{\frac{5}{5}}$ observed a clear peak at a zigzag edge and found no such signal at an armchair edge. The peak in the LDOS is located below the Fermi energy (defined by zero bias voltage) by about $20 \mathrm{meV}$. The inten- 


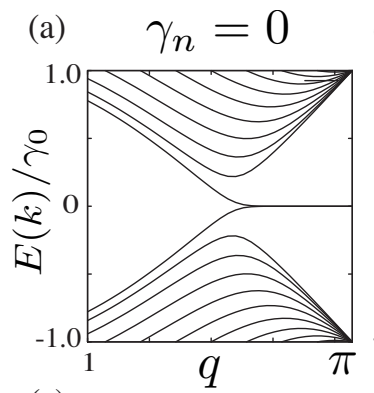

(c)
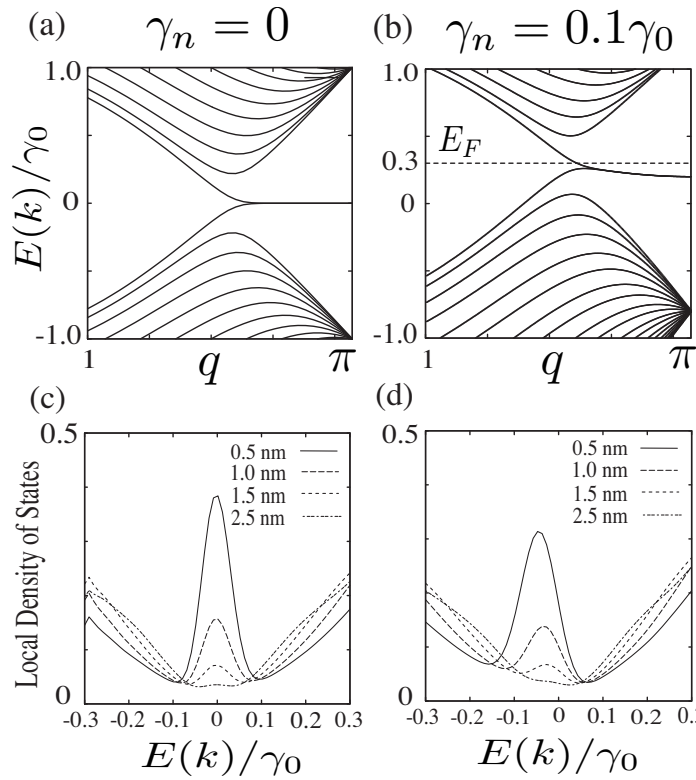

(d)

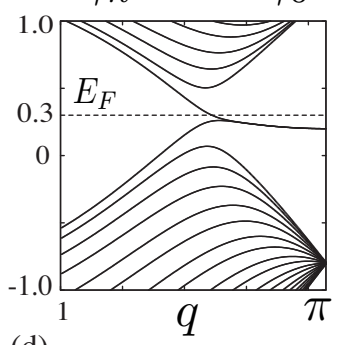

(d)

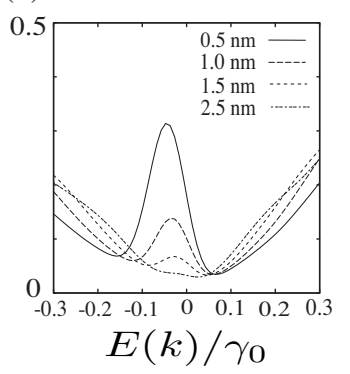

FIG. 2: Energy band structure of zigzag-edge, nano-graphite (a) without the NNN hopping and (b) with the NNN hopping $\left(\gamma_{n}=0.1 \gamma_{0}\right)$. Local density of states at some points from the edges $(0.5-2.5 \mathrm{~nm})(\mathrm{c})$ without the NNN hopping and (d) with the NNN hopping. In (d), $E=0$ is taken as the Fermi energy.

sity of the peak depends on the distance from the edge, which can be attributed to the localized nature of the edge states. Kobayashi et al $\underline{\underline{6}}^{\underline{\underline{n}}}$ observed a similar behavior. In addition, they observed a peak not only at a zigzag edge but also at defect points of an armchair edge, while they found no peak at a homogeneous armchair edge. The important point from our viewpoint of this letter is that the above two experimental groups observed the peak located below the Fermi level by about $10^{-2} \gamma_{0}$. However, this property does not seem to be a common property for edge states. By using the STM and STS, Klusek et al. ${ }^{7.8}$ found peaks of LDOS in the energy range of 20-250 meV above the Fermi level at the edges of circular pits on graphite surface. Although the circular pits have a mixture of zigzag and armchair edge shapes, it is expected that there appear the edge states near (local) zigzag edges in the circular pits since Nakada et al $l^{13}$ showed numerically that localized states appear not only in the zigzag edges but also in edges with other shapes. Such a general edge state is beyond the scope of this letter. It is important to note that samples examined by each experimental group were not prepared under the same condition. Kobayashi et al $\underline{\underline{6}}$ observed the graphene with clear edge structures which were terminated with hydrogen in ultrahigh vacuum (UHV) conditions. Their procedure of sample preparation can exclude functional groups including oxygen which tend to lower the energy of the edge states. On the other hand, there is a possibility that samples used by Niimi et al $\mathrm{s}^{\underline{\underline{n}}}$ and Klusek et $a l^{7.8}$ included such functional groups since their samples were not treated with hydrogen which is activated at high temperatures in UHV conditions.

Although we have demonstrated that the NNN hopping can decrease the energy of the edge state at zigzag edge, there is still a gap between our result, $\Delta E=-\gamma_{n} \approx$ $-10^{-1} \gamma_{0}$, and experimental data,,$\frac{5.6}{\sqrt{3}}-10^{-2} \gamma_{0}$. This gap can be attributed to several physical origins. Among several factors, the Coulomb interaction would give a significant charging energy to the localized edge states. The energy shift due to the NNN hopping depends on the localization length of the edge state and varies from $-\gamma_{n} \leq \Delta E<0$. The most localized edge states have the largest energy shift $\Delta E \approx-\gamma_{n}$; as the localization length becomes longer, the energy shift approaches $\Delta E \approx 0$. Because the Coulomb charging energy is basically inversely proportional to the localization length, the energy shift of the edge state relative to the Fermi level will be reduced. It is noted that the Coulomb interaction is of particular importance from the point of view of the spin polarization $2^{2}$

In summary, we point out that the NNN hopping process decreases the energy of edge states at zigzag edges with respect to the Fermi energy. The energy reduction depends on the localization length of the edge states. The most localized edge states have the largest energy reduction due to the NNN hopping, while the critical states stay on the Fermi level when the sample is sufficiently large. We calculate the energy shift [Eq. [6)] by the first-order perturbation theory, and confirm the result numerically as shown in Fig. [2]

K.S. acknowledges support form the 21st Century COE Program of the International Center of Research and Education for Materials of Tohoku University. S.M. is supported by Grant-in-Aid (No. 16740167) from the Ministry of Education, Culture, Sports, Science and Technology (MEXT), Japan. R.S. acknowledges a Grant-in-Aid (No. 16076201) from MEXT.
* Electronic address: sasaken@imr.tohoku.ac.jp

1 R. Saito, G. Dresselhaus, and M.S. Dresselhaus, Physical Properties of Carbon Nanotubes, (Imperial College Press, London, 1998).

2 M. Fujita, K. Wakabayashi, K. Nakada, and K. Kusakabe, J. Phys. Soc. Jpn. 65, 1920 (1996).
3 D. J. Klein, Chem. Phys. Lett. 217, 261 (1994).

4 S. Okada and A. Oshiyama, J. Phys. Soc. Jpn. 72, 1510 (2003).

5 Y. Niimi, T. Matsui, H. Kambara, K. Tagami, M. Tsukada, and H. Fukuyama, Appl. Surf. Sci. 241, 43 (2005).

${ }^{6}$ Y. Kobayashi, K. Fukui, T. Enoki, K. Kusakabe, and Y. 
Kaburagi, Phys. Rev. B 71, 193406 (2005).

7 Z. Klusek, Z. Waqar, E. A. Denisov, T. N. Kompaniets, I. V. Makarenko, A. N. Titkov, and A. S. Bhatti, Appl. Surf. Sci. 161, 508 (2000).

8 Z. Klusek, Vacuum 63, 139 (2001).

${ }^{9}$ K. Sasaki, S. Murakami, R. Saito, and Y. Kawazoe, Phys. Rev. B 71, 195401 (2005).

10 D. Porezag, Th. Frauenheim, Th. Köhler, G. Seifert, and R. Kaschner, Phys. Rev. B 51, 12947 (1995).

11 Strictly speaking, the Fermi energy depends on the size of the graphite and is located somewhere between $2 \gamma_{n}$ and
$3 \gamma_{n}$. However, if the graphite is sufficiently large $N \gg$ $\gamma_{0} / \gamma_{n}$ the Fermi level can be regarded as $3 \gamma_{n}$ since the number of the bulk states is much larger than that of the edge states.

12 Ge. G. Samsonidze, R. Saito, N. Kobayashi, A. Grüneis, J. Jiang, A. Jorio, S. G. Chou, G. Dresselhaus, and M. S. Dresselhaus, Appl. Phys. Lett. 85, 5703 (2004).

13 K. Nakada, M. Fujita, G. Dresselhaus, and M.S. Dresselhaus, Phys. Rev. B 54, 17954 (1996). 